\title{
Use of intracavernous injection of prostaglandin E1 for neuropathic erectile dysfunction
}

\author{
I H Hirsch MD,${ }^{1} \mathrm{R}$ L Smith MD,${ }^{1} \mathrm{M}$ B Chancellor MD,${ }^{1} \mathrm{D} \mathrm{H}$ Bagley $\mathrm{MD},{ }^{1} \mathrm{~J}$ Carsello \\ $\mathrm{RN},{ }^{1} \mathrm{~W}$ E Staas Jr MD ${ }^{2}$
}

\author{
${ }^{1}$ Department of Urology, ${ }^{2}$ Department of Rehabilitation Medicine, Jefferson Medical \\ College, Philadelphia, PA, USA
}

The administration and suitability of intracavernous PGE-1 in men with neuropathic erectile dysfunction is reported herein. Twenty-seven men with neuropathic erectile dysfunction (SCI, 14; multiple sclerosis, 7; discogenic disease, 6) were evaluated and treated with intracavernous PGE-1. An average of 3.2 office sessions were required to learn adequate self-injection technique and determine optimal dosage requirements. Initial dosage for SCI men was $2.5 \mu \mathrm{g}$ and increased in $2.5 \mu \mathrm{g}$ increments to a mean maintenance dose of $6.2 \mu \mathrm{g}$. Quarterly monitoring up to 28 months demonstrated satisfactory erectile rigidity and duration of erection in all patients electing to pursue home administration of PGE-1. During this interval, over $40 \%$ of patients dropped out of the treatment program. No priapism or changes in serum chemistries, $\mathrm{CBC}$, or platelets were observed during this period. Corporal fibrosis although not palpable, was detected subclinically by penile ultrasound in two men. This study confirms the safety and efficacy of self-administered intracavernous PGE-1 for neuropathic impotence. However, because of a significant rate of voluntary cessation, patients should be counseled regarding the full range of therapeutic alternatives to intracavernous therapy.

Keywords: neurological diseases; impotence; intracavernous therapy; prostaglandin E1.

\section{Introduction}

During the past decade intracavernous therapy has been investigated and widely utilized as an alternative to conventional treatment methods (penile prosthesis and vacuum erection devices). Vasoactive agents such as papaverine, phentolamine and prostaglandin E1 (PGE-1) used either as single agents or in combination have been the drugs of choice. Prostaglandin E-1 has received increasing attention because papaverine has been associated with such complications as corporal fibrosis, priapism and hepatic dysfunction. While intracavernous therapy can address erectile dysfunction of

Correspondence to Irvin $\mathrm{H}$ Hirsch $\mathrm{MD}$, Jefferson Medical College, Department of Urology, 1025 Walnut Street, 11th floor College Bldg, Philadelphia, PA 19107, USA. virtually any cause, it demonstrates the highest success rates in patients with a neurological cause. ${ }^{1}$ Studies reporting the utility of intracavernous therapy among men with spinal cord injury and other neurological sources focus primarily on the intracavernous agents of papaverine monotherapy or in combination with phentolamine. ${ }^{2}$ By contrast relatively little information has been accumulated reporting the clinical experience with prostaglandin E- 1 in the setting of neuropathic erectile dysfunction. This study reports our experience with intracavernous prostaglandin $\mathrm{E}-1$ as a monotherapeutic agent for the treatment of neuropathic erectile dysfunction.

\section{Patients and methods}

Initial evaluation, including medical and sexual histories, physical examination, and 
penile brachial systolic index, was performed in patients with neuropathic erectile dysfunction. Serum chemistries, testosterone and prolactin were routinely measured. When indicated nerve conduction studies, and nocturnal penile tumescence and duplex Doppler evaluation were carried out. In 41 men with neuropathic impotence diagnostic intracavernous PGE-1 injection was performed. Of these 27 expressed a preference for continued treatment by self administered home therapy. The etiologic categories of neurological disease included spinal cord injury, 14; multiple sclerosis, 7; and disc disease, 6. In these groups the mean duration of erectile dysfunction was 8 , 9,7 years, respectively. Initial dose titration began at $2.5 \mu \mathrm{g}$ of PGE-1. Office-based titration doses and home maintenance doses were monitored during quarterly follow up visits up to 28 months. Subjects were instructed on the proper technique of self injection using a 27 gauge needle. Dose escalation determined by the erectile response achieved during the office dose titration phase, was performed by dose increments of $2.5 \mu \mathrm{g}$.

Over the 18 month follow up interval quarterly complete blood count (CBC), measurement of serum chemistry and penile ultrasound were performed to study outcome with special reference to local and systemic complication, and assessment of erectile response.

\section{Results}

While all groups presented for treatment with a similar duration of disease, SCI men were relatively younger (Table I). Mean maintenance doses were $6.2 \mu \mathrm{g}, 6.8 \mu \mathrm{g}$, and $8.2 \mu \mathrm{g}$ for men with SCI, multiple sclerosis and disc disease, respectively. Of the 27 patients enrolled in self administer intracavernous therapy, 12 patients discontinued therapy when followed up to 28 months. Within each category the rate of voluntary cessation was $43 \%, 43 \%$ and $50 \%$ for groups of SCI, multiple sclerosis and disc disease, respectively. There were multiple reasons for discontinuation, including loss of partner, loss of interest, urinary diversion, adrenal tumor, financial, and substance abuse (Table II). No patients discontinued therapy because of inadequate erectile response or pain. All patients completing the protocol reported excellent penile rigidity on standardized home assessment questionnaires. Quarterly evaluation failed to disclose any systemic or local complication. Although no plaques were palpable, two patients (multiple sclerosis, disc disease) were found to have subclinical corporal fibrosis as evidenced by new

Table I Patient population

\begin{tabular}{lccccc}
\hline & $\begin{array}{c}\text { No. } \\
\text { evaluated }\end{array}$ & $\begin{array}{c}\text { No. } \\
\text { enrolled }\end{array}$ & $\begin{array}{c}\text { Age } \\
\text { range }\end{array}$ & $\begin{array}{c}\text { Average } \\
\text { Age }\end{array}$ & $\begin{array}{c}\text { Duration of erectile } \\
\text { dysfunction (yrs) }\end{array}$ \\
\hline Spinal cord injury (SCI) & 21 & 14 & $22-39$ & 31.5 & 8 \\
Multiple sclerosis (MS) & 10 & 7 & $31-51$ & 43 & 9 \\
Disc disease & 10 & 6 & $36-64$ & 46.6 & 7 \\
\hline
\end{tabular}

Table II Voluntary cessation and reasons

\begin{tabular}{lcccl}
\hline & Enrolled & Remained & $\begin{array}{c}\text { \% Drop } \\
\text { out }\end{array}$ & Reasons \\
\hline Spinal cord injury (SCI) & 14 & 8 & 43 & $\begin{array}{l}\text { Urinary diversion, adrenal } \\
\text { tumor, loss of interest, insurance } \\
\text { difficulties }\end{array}$ \\
$\begin{array}{l}\text { Multiple sclerosis (MS) } \\
\text { Disc disease }\end{array}$ & 7 & 4 & 43 & $\begin{array}{l}\text { Interactive loss of interest } \\
\text { Loss of partner, undermined, } \\
\text { substance abuse }\end{array}$ \\
\hline
\end{tabular}


echogenic foci noted on sequential penile ultrasound scanning. In addition to the absence of local complications these patients did not demonstrate any systemic alterations by periodic assessment of blood pressure, liver function tests, $\mathrm{CBC}$ and routine serum chemistries.

\section{Discussion}

A significant portion of the estimated 10 million impotent men in the USA have a solitary or contributory neuropathic etiology for their dysfunction. In particular, patients with central nervous system pathology such as multiple sclerosis, spinal cord injury and disc disease are especially prone to this complication. Normal erectile physiology requires compliant corporal smooth muscle and intact neurovascular function. Cholinergic input from the sacral outflow and modulated input from thoracolumbar spinal centers cause corporal smooth muscle relaxation and corporal sinus engorgement as the mechanism or erection. The thoracolumbar outflow is believed to be responsible for psychogenic erection while the sacral cord segment creates a reflex erection response to tactile stimulation. ${ }^{3}$

Spinal cord injured men have a varying degree of erectile function depending on the degree and level of injury. Those individuals with sacral sparing lesions above T10-12 often maintain reflexogenic erections in response to genital stimulation. ${ }^{4}$ Conversely, men with lower motor neuron injuries may achieve erections with visual sensory stimulation via intact thoracolumbar outflow. ${ }^{5}$ This psychogenic erection, however, has been described as poorly sustained and nonrigid ${ }^{6}$ limiting the possibility of adequate intromission.

Multiple sclerosis presents with a multitude of symptoms frequently including male sexual dysfunction reported in $47-62 \%$ of patients. ${ }^{7}$ Erectile dysfunction is this setting results from abnormal or absent saltatory nerve conduction. Plaque formation characterized by loss of oligodentrocytes and proliferation of fibrous astrocytes may occur at multiple levels in the central nervous system. These aberrations in nerve conduction velocity within the autonomic nervous system are likely to be the mechanism responsible for the symptoms of erectile dysfunction in multiple sclerosis. ${ }^{8}$

Disc protrusion and herniation have been the cause of sacral and cauda equina compression and variable degrees of associated erectile dysfunction. ${ }^{9}$ In addition ejaculatory, voiding and bowel dysfunction may be observed $^{10}$ if sacral outflow is affected. Nocturnal penile tumescence studies have demonstrated the presence of nocturnal erections in patients with discogenic disease. This interesting finding suggests that neuropathic impotence is a variable phenomenon with variable degrees on intensity. The presence of nocturnal erections in the setting of lower motor neuron dysfunction may be explained by the intact residual corticospinal outflow that produces nocturnal erections.

Prostaglandin E1 (PGE-1) has been shown to effectively relax corporal smooth muscle in in vitro studies. ${ }^{11}$ This property forms the basis for its clinical application in diagnostic protocols and therapeutic regimens for erectile dysfunction. Clinical studies of intracavernous PGE-1 have established a far lower incidence of priapism, hepatic dysfunction and local fibrotic changes compared to conventional intracavernous agents (papaverine/phentolamine). ${ }^{12}$

The present study demonstrates that intracavernous therapy with prostaglandin $\mathrm{E} 1$ is an effective therapeutic modality for the short term rehabilitation of patients with neuropathic erectile dysfunction. The consistent and complete response of such patients allows for prompt rehabilitation of erectile dysfunction with minimal systemic and local adverse events. The categories of neurological disease studied herein represent suitable clinical settings for intracavernous therapy. These men are generally younger than patients with vascular etiology for erectile dysfunction and therefore have less age-related vascular disease. Providing intracorporal vasoactive agents by direct intracavernous injection bypasses the neuropathic requirements for erection to yield a satisfactory erectile response. Adequate penile rigidity can be achieved at relatively lower doses than cohorts with vascular 
etiology. However, because of the relatively high voluntary cessation rate noted with intracavernous therapy, long term therapeutic measures should be considered in patients with neuropathic erectile dysfunction.

Thus far, complications such as infection and fibrotic plaques have not been reported with PGE-1. In comparative clinical reports, PGE-1 had a superior outcome compared to papaverine/phentolamine and was associated with a substantially lower incidence of priapism. ${ }^{13}$ The incidence of priapism in men with neuropathic erectile dysfunction ranges from $10 \%$ to $25 \% ., 14$
Owing to the relatively functional integrity of penile hemodynamics in these men, the complication of priapism and prolonged erection must be monitored carefully in this group. Our study demonstrates the absence of this complication in our patients with neuropathic erectile dysfunction both in the office-based dose titration phase, as well as in the home-based maintenance dosing interval.

We attribute our favorable finding to the agent itself as PGE-1 has been noted in our experience and in a variety of studies to have the advantage of minimal complications of priapism.

\section{References}

1 Sidi AA, Cameron JS, Duffy LM, Lange PH (1986) Intracavernous drug induced erections in the management of male erectile dysfunction: experience with 100 patients. J Urol 135: 704-708.

2 Wyndaele JJ, DeMeyer JM, DeSy WA, Claessens, H (1986) Intracavernous injection of vaso-active drugs, an alternative for treating impotence in spinal cord injury patients. Paraplegia 24: 271-275.

3 Krane RJ, Goldstein I, Saenz de Tejada I (1989) Impotence. N Engl J Med 321: 1648-1653.

4 Comarr AE (1971) Sexual concepts in traumatic cord and cauda equina lesions. J Urol 106: $375-378$.

5 Bors E, Comarr EA (1980) Neurological disturbances of sexual function with special references to 529 patients with spinal cord injury. Urol Surv 10: 191-214.

6 Chapelle PA, Durand J, Lacert P (1980) Penile erection following complete spinal cord injury in men. Br J Urol 52: 216.

7 Valleroy ML, Kraft HG (1984) Sexual dysfunction in multiple sclerosis. Arch Phys Med Rehabil 65: 125-128.

8 Goldstein I, Siroky MB, Sax DS, Krane RJ (1982) Neurologic abnormalities in multiple sclerosis. J Urol 28: $541-545$.

9 O'Laoire SA, Crockard HA, Thomas DG (1981) Prognosis for sphincter recovery after operation for cauda equina compression owing to lumbar disc prolapse. BMJ 282: 1852-1854.

10 Velcek D (1989) Discogenic impotence. Int J Impotence Res 1: 95-113.

11 Hedlund H, Andersson KE (1985) Contraction and relaxation induced by some prostanoids in isolated human penile tissue and cavernous artery. J Urol 134: 1245-1250.

12 Juneman KP, Alkin P (1989) Pharmacotherapy for erectile dysfunction: A review. Int J Impotence Res 1: 71-94.

13 Earle CM, Keogh EJ, Wisniewski SZ et al (1990) Prostaglandin E1 therapy for impotence comparison with papaverine. J Urol 143: 57-59.

14 Bodner DR, Lindan R, Leffler E et al (1987) The application of intracavernous injection of vaso-active medication for erection in men with spinal cord injury. J Urol 138: 310-311. 